\title{
Coxsackievirus and adenovirus receptor (CAR) mediates atrioventricular-node function and connexin 45 localization in the murine heart
}

\author{
Byung-Kwan Lim, ${ }^{1}$ Dingding Xiong, ${ }^{1}$ Andrea Dorner, ${ }^{2}$ Tae-Jin Youn, ${ }^{3}$ Aaron Yung, ${ }^{1}$ \\ Taylor I. Liu, ${ }^{1}$ Yusu Gu, ${ }^{1}$ Nancy D. Dalton, ${ }^{1}$ Adam T. Wright, ${ }^{4}$ Sylvia M. Evans, ${ }^{5}$ Ju Chen, ${ }^{1}$ \\ Kirk L. Peterson, ${ }^{1}$ Andrew D. McCulloch, ${ }^{4}$ Toshitaka Yajima, ${ }^{1}$ and Kirk U. Knowlton ${ }^{1}$ \\ 1Department of Medicine, University of California San Diego, Division of Cardiology, La Jolla, California, USA. \\ ${ }^{2}$ Charité-University Medicine, Campus Benjamin Franklin, Center 11, Cardiology and Pneumology, Berlin, Germany. \\ ${ }^{3}$ Seoul National University BunDang Hospital, BunDang, Gyeunggido, Republic of Korea. ${ }^{4}$ Department of Bioengineering and \\ ${ }^{5}$ Skaggs School of Pharmacy, University of California San Diego, La Jolla, California, USA.
}

\begin{abstract}
The coxsackievirus and adenovirus receptor (CAR) is a transmembrane protein that belongs to the family of adhesion molecules. In the postnatal heart, it is localized predominantly at the intercalated disc, where its function is not known. Here, we demonstrate that a first degree or complete block of atrioventricular (AV) conduction developed in the absence of CAR in the adult mouse heart and that prolongation of AV conduction occurred in the embryonic heart of the global CAR-KO mouse. In the cardiac-specific CAR-KO (CAR-cKO) mouse, we observed the loss of connexin 45 localization to the cell-cell junctions of the AV node but preservation of connexin 40 and 43 in contracting myocardial cells and connexin 30.2 in the AV node. There was also a marked decrease in $\beta$-catenin and zonula occludens-1 (ZO-1) localization to the intercalated discs of CAR-cKO mouse hearts at 8 weeks before the mice developed cardiomyopathy at 21 weeks of age. We also found that CAR formed a complex with connexin 45 via its PSD-95/DigA/ZO-1-binding (PDZ-binding) motifs. We conclude that CAR expression is required for normal $\mathrm{AV}$-node conduction and cardiac function. Furthermore, localization of connexin 45 at the AV-node cell-cell junction and of $\beta$-catenin and ZO-1 at the ventricular intercalated disc are dependent on CAR.
\end{abstract}

\section{Introduction}

The coxsackievirus and adenovirus receptor (CAR) was initially identified as a common receptor of the viruses for which it was named $(1,2)$. It is expressed in various organs including the heart and is indispensable for normal embryonic development (3-5). In the adult heart, there is considerable data on the role of CAR as a viral receptor in the pathogenesis of viral myocarditis; however, the physiological role of CAR in the adult heart is less clear.

CAR is a transmembrane protein with 2 extracellular immunoglobulin domains that belongs to the family of intercellular adhesion molecules. The extracellular region of CAR is thought to bind to another CAR molecule on an adjacent cell as an antiparallel homodimer (6). The intracellular domain of CAR contains a carboxyterminal hydrophobic peptide that interacts with PSD-95/ DigA/ZO-1 (PDZ; ZO-1, zonula occludens-1) domain-containing proteins such as ZO-1 $(7,8)$. During embryonic development, CAR is expressed by E7, and in neonatal mice, CAR is expressed at relatively high levels in the heart (3). After 1 week of age, the majority of CAR is localized at the myocyte-myocyte junctional complex known as the intercalated disc (9).

Nonstandard abbreviations used: APD, action potential duration; AV, atrioventricular; CAR, coxsackievirus and adenovirus receptor; CAR-cKO, cardiac-specific CAR KO; FHL2, 4.5 LIM domain protein 2; HCN4, hyperpolarization-activated, cyclic nucleotide-gated cation channels 4 ; $\alpha$-MHC-CRE, $\alpha$-myosin heavy chain-CRE; PDZ, PSD-95/DigA/ZO-1; SA, sinoatrial; ZO-1, zonula occludens-1.

Conflict of interest: The authors have declared that no conflict of interest exists. Citation for this article: J. Clin. Invest. 118:2758-2770 (2008). doi:10.1172/JCI34777.
Of the conduction system components, the atrioventricular (AV) node has a unique role in delaying conduction of the electrical impulse from the atria to the ventricle. When AV-node function is impaired, there is an abnormal delay in or absence of conduction from the atria to the ventricles. The gap junctions that are found in the cell-to-cell contacts between AV-nodal cells and in the intercalated discs of cardiac myocytes are important for impulse propagation $(10,11)$. There are 4 principal connexins that are found in the gap junctions of the adult heart (connexins 40, 43, 45, and 30.2) (12). Connexins 45 and 30.2 are the predominant connexins that are known to be expressed in regions of the AV-nodal tissue $(13,14)$.

In this manuscript, we hypothesize that disruption of CAR affects the function of intercalated discs in the adult heart and that it may affect gap junctions in the $\mathrm{AV}$ node and the intercalated discs of cardiac myocytes. We found that cardiac-specific KO of CAR led to abnormal propagation of electrical conduction in the AV node, which was associated with decrease in connexin 45 expression on the cell membrane. At later time points, the mice developed cardiomyopathy associated with focal disorganization of the intercalated disc ultrastructure and loss of localization of $\beta$-catenin and $\mathrm{ZO}-1$ in the cardiac myocytes. Colocalization of CAR, ZO-1, and connexin 45 in a protein complex requires the PDZ-binding motif on CAR and connexin 45 . These data demonstrate the importance of CAR in the normal adult heart.

\section{Results}

Generation of cardiac-specific CAR-KO mice. A floxed CAR construct was generated by inserting loxP sites just before and just after the 
A

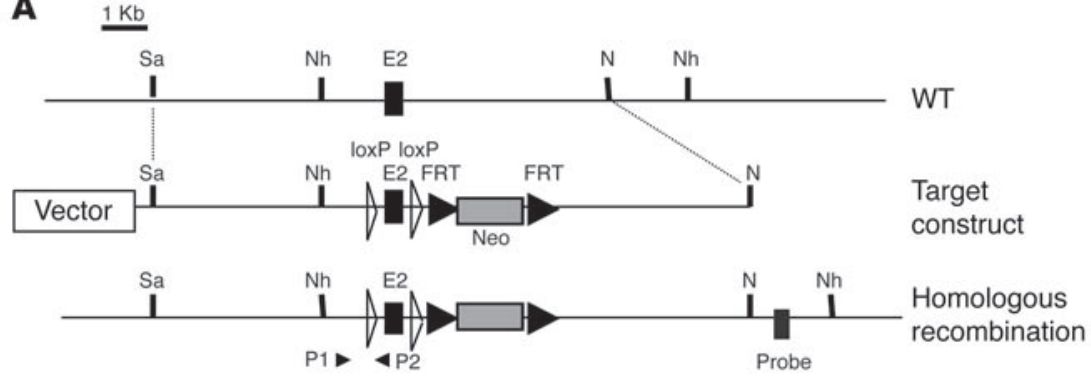

B

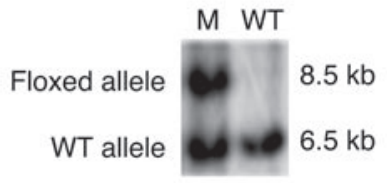

D

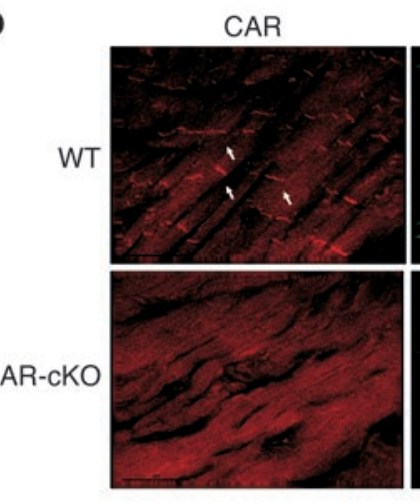

C
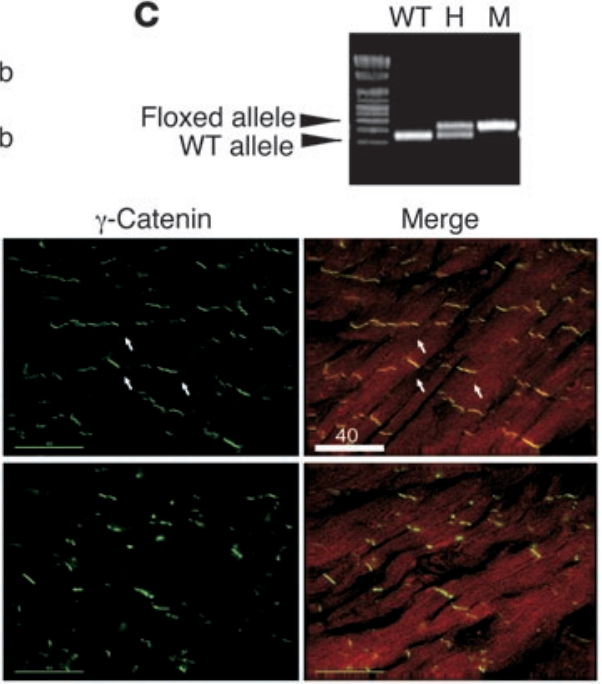

\section{Figure 1}

Generation of CAR-cKO mice. (A) Targeting strategy for deletion of $C A R$. Exon 2 of the murine $C A R$ gene was flanked by loxP sites, and a neomycin (Neo) resistance cassette was inserted downstream, flanked by FRT sites. Sa, Sall; Nh, Nhel; N, Notl. (B) Genomic Southern blot analysis of mutant floxed allele (M) or WT ES cells using probe shown in panel $A$, after digestion of genomic DNA with Nhel. (C) PCR of WT, floxed heterozygous $(H)$, or homozygous $(M)$ mutant mice from extracted mouse-tail genomic DNA using primers P1 and P2. (D) Disruption of CAR expression in WT or CAR-cKO mice as measured by immunofluorescent staining and immunoblotting using an antibody specific for CAR (red) and $\gamma$-catenin (green) as marker of the cell-cell junction. The typical intercalated disc staining for CAR is noted in the WT mice (arrows) but absent in the CAR-cKO mice. Scale bar: $40 \mu \mathrm{m}$.

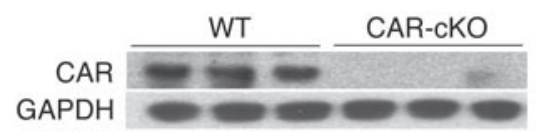

second exon that contains the ATG start codon (Figure 1, A-C). Mice containing the genetically modified CAR allele were bred with transgenic mice that harbored the cardiac-specific $\alpha$-myosin heavy chain-CRE ( $\alpha$-MHC-CRE) transgene (15). Immunoblot and immunofluorescent staining for CAR in 20-week-old mice confirmed that $70 \%-90 \%$ of CAR was deleted from the intercalated discs of cardiac myocytes in CARflox/flox $\alpha$-MHC-CRE mice (CAR-cKO). However, $\gamma$-catenin localization was not altered (Figure 1D). $C A R^{\text {flox/flox }}$ or $C A R^{\text {flox/+ }}$ littermate mice were used as a control (WT).

Abnormal AV conduction in the CAR-cKO mice. Electrocardiograms in lightly anesthetized 4- to 5-week-old mice demonstrated normal AV conduction in the wild-type mice but evidence of complete AV block in the CAR-cKO mice (Figure 2A). High-resolution signal average of 100 ventricular depolarizations demonstrated no significant difference in the QRS morphology between the WT and CAR-cKO mice; however, because of the dissociation between the atrial and the ventricular depolarizations, an averaged $\mathrm{P}$-wave was not detectable in the CAR-cKO mice (Figure 2A). The QRS duration was $13.5 \pm 0.56$ vs. $14.3 \pm 0.54 \mathrm{~ms}(P=\mathrm{NS})$, and the RR intervals were $127.3 \pm 12.6$ vs. $97.4 \pm 5.9 \mathrm{~ms}(P<0.05)$ in WT and CAR-cKO mice, respectively (mean $\pm \mathrm{SD} ; n=3$ mice in each group). Given these results, we performed telemetric ECG analysis in awake, conscious mice at 8 weeks of age. All WT mice had normal sinus rhythm with normal AV conduction. However, all CAR-cKO mice had abnormal AV conduction. In 3 of 4 mice, the AV block was complete at some point during the recording. Complete heart block was the only rhythm in 1 mouse and was present over $75 \%$ of the time in another. The other 2 mice had first degree AV block over $90 \%$ of the time, with scattered episodes of complete AV block in 1 of these mice (Figure $2 \mathrm{~B}$ ). The PR interval was $36.5 \pm 1.9$ in the 4 WT mice compared with $53.9 \pm 2.7$ in the 2 CAR-cKO mice that had predominantly first degree AV block (mean $\pm \mathrm{SD}, P<0.01 ; n=96$ randomly selected PR intervals for WT and 48 randomly selected PR intervals for CAR-cKO). Second-degree AV block was not detected during any of the recordings. Intravenous dobutamine infusion of 0.75 to $4 \mu \mathrm{g} / \mathrm{kg} / \mathrm{min}$ did not cause a significant change in the AV dysfunction in 3 mice evaluated (data not shown).

Normal atrial and ventricular action potential propagation in CAR-cKO mice. Given the electrocardiographic abnormalities in AV conduction, function of the intraatrial and intraventricular conduction system was evaluated in the presence and absence of CAR using optical mapping of intrinsic epicardial action potential propagation in isolated hearts from 4- to 6-week-old WT $(n=7)$ and CAR-cKO $(n=6)$ mice. Analysis of the timing and pattern of atrial depolarization demonstrated that the atrial impulse originated at or near the area of the sinoatrial (SA) node and that there was little difference in the timing or general pattern of intrinsic activation of the atria between WT and CAR-cKO mice. The time required for depolarization of the atria during an intrinsic beat as measured from the anterior optical map was $6.3 \pm 1.6$ vs. $7.7 \pm 2.4 \mathrm{~ms}(P=\mathrm{NS})$, and depolarization from the posterior view was $7.3 \pm 3.0$ vs. $8.6 \pm 3.6(P=\mathrm{NS})$ for WT and $\mathrm{CAR}-\mathrm{cKO}$ mice, respectively (mean $\pm \mathrm{SD}$ ) (Figure $2 \mathrm{C}$ ). 
A

Signal average of 100 beats

WT

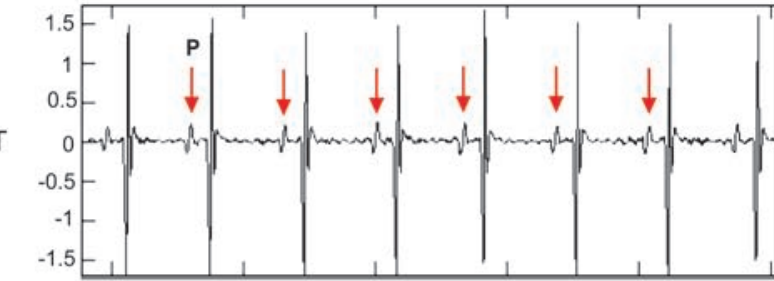

Signal average of 100 beats

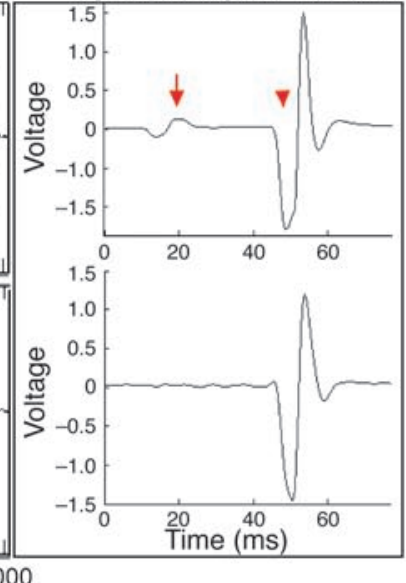

CAR-CKO

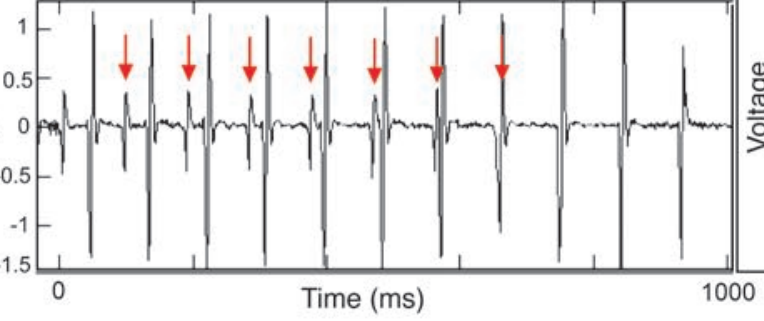

B

WT

CAR-cKO
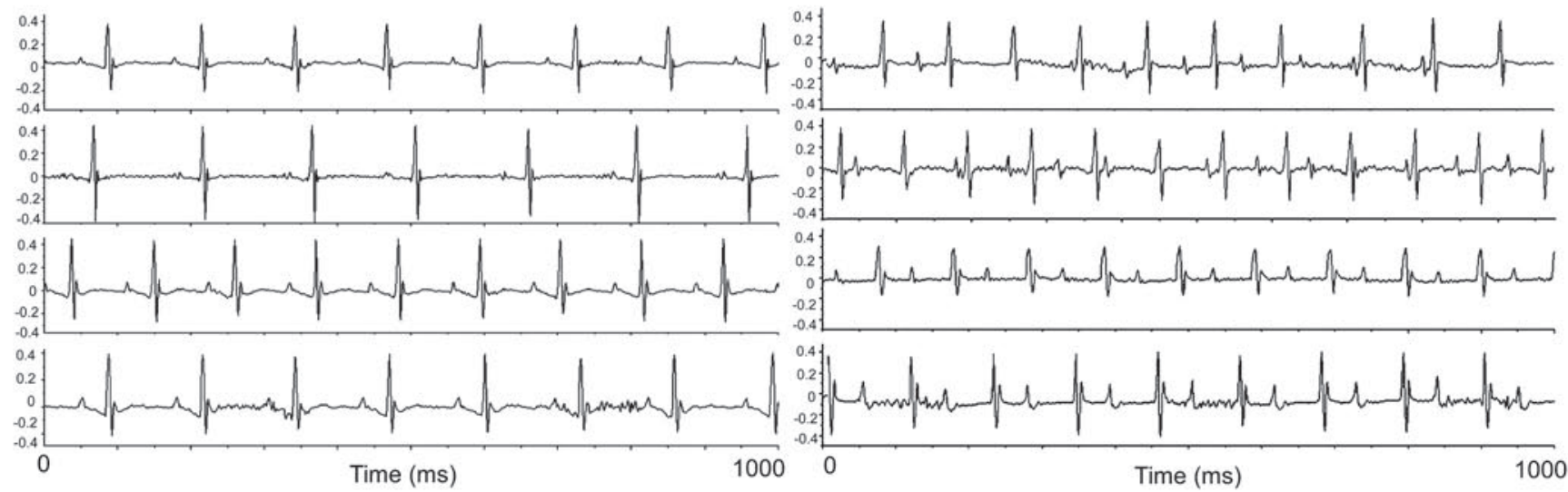

C
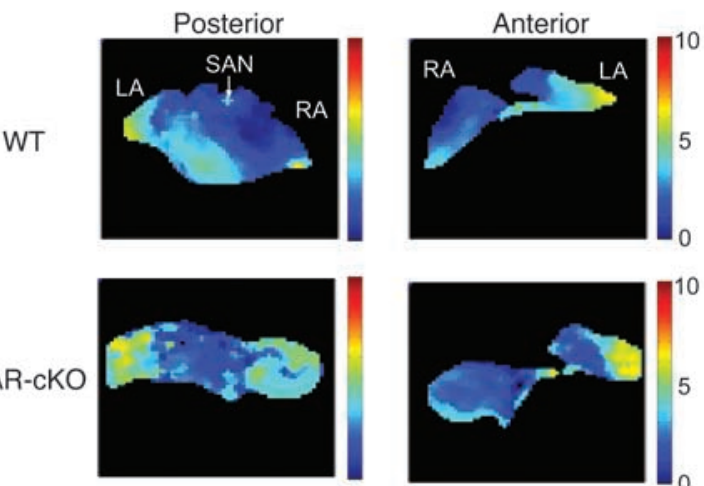

D

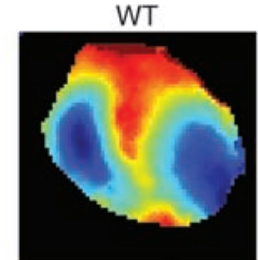

CAR-cKO
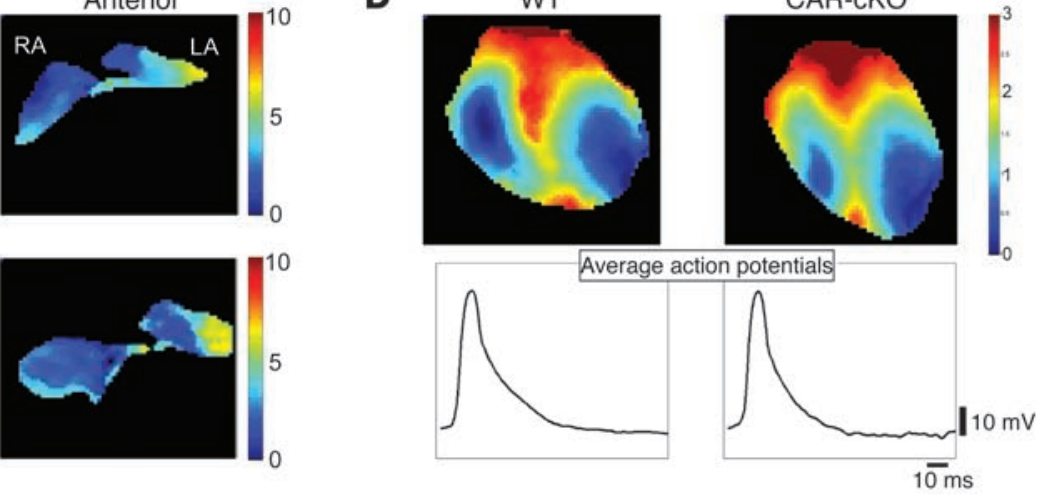

Conduction velocity

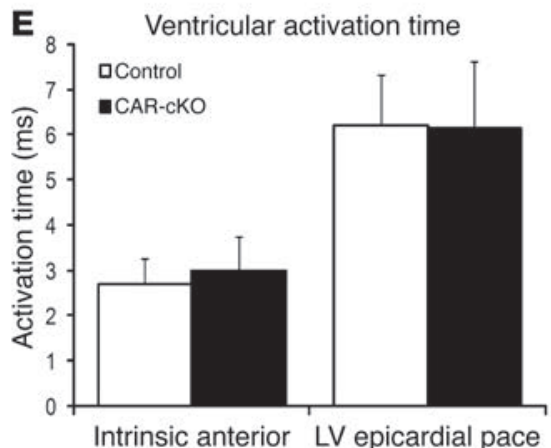
during ventricular pacing

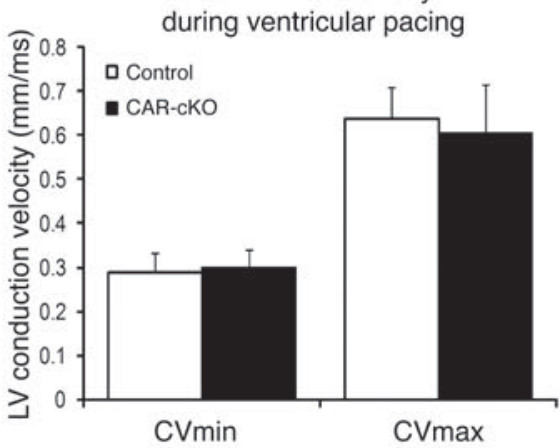

CVmin
$\mathrm{LV}$ epicardial pacing $(\mathrm{BCL}=150 \mathrm{~ms})$

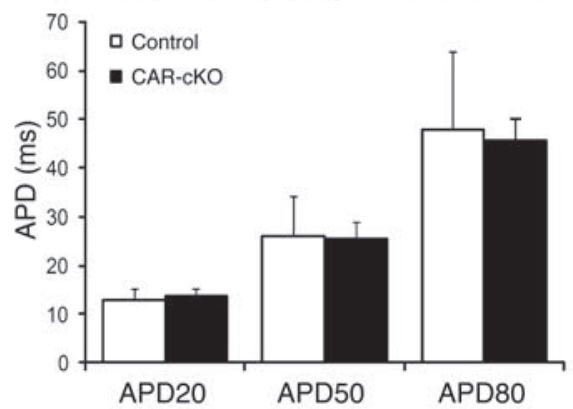




\section{Figure 2}

AV-node block in CAR-cKO mice (A) Electrocardiograms obtained from lightly anesthetized WT and CAR-cKO 4- to 5-week-old mice demonstrated complete AV dissociation in CAR-cKO mice. P (arrow) indicates atrial depolarizations ( $\mathrm{P}$-wave). Right panel shows representative signal averages of 100 beats obtained using high-resolution ECG recording in WT and CAR-cKO mice. P-wave (arrow) and QRS complex (arrowhead) are shown in a WT mouse. The P-wave is not present in the CAR-cKO tracing because it was not associated with the QRS complex and was lost in the signal averaging. (B) Telemetric ECG analysis in conscious mice. All WT mice had normal AV conduction. CAR-cKO mice had either complete AV block (top 2 tracings) or first degree AV block (bottom 2 tracings). None of the CAR-cKO mice had normal AV conduction. (C and D) Optical mapping studies in CAR-cKO mice. The activation maps showed the pattern of intrinsic anterior or posterior activation of isolated atria (C) and epicardial intrinsic activation of isolated heart ventricles (D). There was no significant difference in the activation propagation pattern or averaged action potentials over several beats in the atria or ventricles of WT versus CAR-cKO mice. LA, left atrium; SAN, sinoatrial node; RA, right atrium. (E) Ventricular activation times from the anterior view with an intrinsic depolarization and LV epicardial pacing is shown (left panel). The conduction velocity was not different between WT and CAR-cKO mice (middle panel). APD from LV epicardial pacing was also not different (APD20, APD50, and APD80) (right panel). $P=$ NS between all groups; WT $(n=7)$ vs. CAR-cKO $(n=6)$; mean \pm SD. BCL, basal cycle length.

In addition to the normal atrial depolarization, there was no significant difference in the time required for activation of intrinsic ventricular depolarization based on the optical map or the pattern of activation of the ventricles between WT and CAR-cKO mice (Figure 2, D and E). This included evidence of nearly simultaneous depolarization of both the RVs and LVs through both bundle branches. In addition, average action potential patterns did not differ significantly between WT and CAR-cKO mice (Figure 2D). Furthermore, there was no difference in anterior ventricular activation times between WT and CAR-cKO mice during intrinsic heart rhythms or during LV epicardial pacing. In addition, there was no significant difference in action potential duration (APD) or conduction velocity in hearts following epicardial pacing of the ventricle (Figure 2E). However, there was evidence of impaired AV conduction as manifested by complete AV block in $100 \%$ of the hearts isolated from CAR-cKO mice $(n=6)$.

$A V$ block in global CAR-KO embryos. In order to determine whether there were conduction system abnormalities during development in global CAR-KO embryos, we evaluated AV conduction in global CAR-deficient mice we had previously generated by gene targeting as described in Methods (Figure 3, A and B). To verify that the embryos were deficient for both CAR alleles, we performed Southern blot on DNA isolated from the embryo yolk sac or embryo tail (Figure 3C). Complete disruption of CAR protein expression was confirmed by Western blot analysis (Figure 3C).

Similar to previously published results $(4,5)$ from other laboratories, we found that the CAR-deficient embryos generally died between E11.5 and E12.5 (data not shown). In addition, we found that some of the CAR-deficient embryos had evidence of hemorrhage and a large pericardial effusion at E10.5-E11.5. Histologic analysis of the heart of the CAR-deficient embryos failed to demonstrate reproducibly detectable structural defects, hypertrophy, or ventricular wall thinning.

Since it is not currently feasible to measure the electrocardiogram in a mouse embryo in utero, the flow of blood in the heart of embryos in lightly anesthetized pregnant females was assessed using
Doppler echocardiography. The onset of blood flow from the atrium to the LV during atrial contraction, A-wave, served as a marker for the beginning of atrial depolarization. Similarly, the onset of blood flow through the aortic valve, aortic outflow, served as a marker for the beginning of ventricular depolarization. By measuring the interval between the beginning of the A-wave and the beginning of the aortic outflow, the delay in conduction from the atria to the ventricle was quantitated as a surrogate of the PR interval, the electrocardiographic measure of the time from the beginning of atrial depolarization to the beginning of ventricular depolarization (Figure 3D). PR interval was evaluated in E10.5, E11.5, and E12.5 embryos. The mean PR intervals in $C A R^{-/-}$embryos were significantly different from those of $C A R^{+/+}$and $C A R^{+/-}$mice (Figure 3D). PR intervals in $C A R^{+/+}$embryos did not change between E10.5 and E11.5, but the mutant hearts had stopped beating by E12.5. 100\% of the $C A R^{-/-}$mice had prolonged PR intervals, consistent with first-degree AV block.

$C A R$ expression in the $A V$-conduction system. To determine whether CAR was expressed in the AV-conduction system, hyperpolarization-activated, cyclic nucleotide-gated cation channels 4-H2B (HCN4-H2B) GFP knockin mice were examined. In these mice, nuclear GFP expression marks the conduction tissue (16). We assessed GFP and CAR immunostaining in the area of the AV node. The most intense staining for CAR in GFP-positive cells was at cell-cell junctions (Figure 4, A-E).

To assess the overall structure of the AV node, CAR-cKO and WT mice were stained with acetylcholinesterase, a marker of cardiac conduction cells. This suggested that the AV-node structures in CAR-cKO mice were similar to those in WT mice (Figure 4F).

Given the importance of connexins $45,30.2$, and 40 in the cardiac conduction system, the level of their expression was evaluated in CAR-cKO hearts and compared with that in WT mice. Immunoblot demonstrated a marked reduction in connexin 45 and an increase in connexin 30.2 from whole heart and an increase in connexin 40 from atria in the CAR-cKO mice. The amount of $\mathrm{HCN} 4$ was unchanged in CAR-cKO mice (Figure 5A).

Localization of connexin 45 was assessed in the AV-nodal cellcell junction in WT and CAR-cKO mice. HCN4 protein localized to the AV node in both WT and CAR-cKO mice. In the WT mice, CAR was expressed in the same cells as HCN4 and was colocalized with connexin 45 at the cell-cell junctions of the AV node. In addition, CAR was colocalized with $\gamma$-catenin as a cell-cell junction marker in the AV node. However, in CAR-cKO mice, CAR localization was disrupted in AV-nodal cells that expressed HCN4. Disruption of CAR expression led to loss of connexin 45 localization in the AV-node cell-cell junctions, but $\gamma$-catenin localization was not disrupted (Figure 5, B-CC).

Abnormalities of cardiac intercalated disc proteins in CAR-cKO mouse ventricular myocytes. To better understand the role of CAR in cardiac cellcell junctions and to determine whether the abnormalities described above are unique to the conduction system, the levels of several intercalated disc proteins were compared at 8 weeks of age in the ventricle of WT versus CAR-cKO mice. At this point in time, ventricular function was normal (see below). We found that expression and localization of adherens junction (pan cadherin, $\alpha$-catenin, and vinculin), desmosome (desmoplakin), and gap junction (connexin 43) proteins were not markedly different between WT and CAR-cKO mice as shown by immunoblot. However, there were reduced amounts of $\beta$-catenin and variably decreased levels of ZO-1 (Figure 6A).

Immunofluorescent staining of the ventricular myocardium of the CAR-cKO mice demonstrated that pan-cadherin, vinculin, des- 
A AT allele
Target
construct
Targeted
allele

B

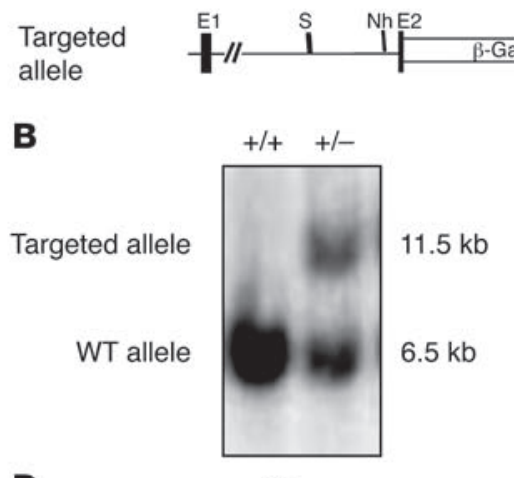

D

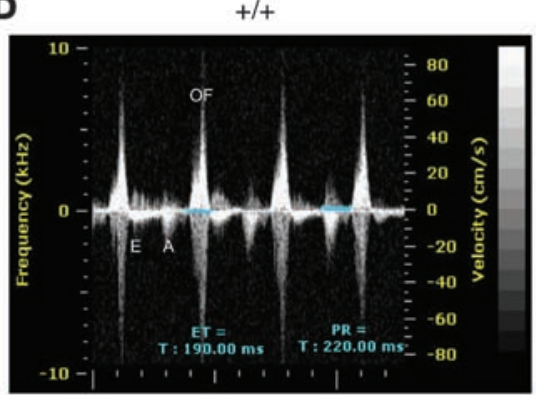

0 $\underline{1 \mathrm{~kb}}$

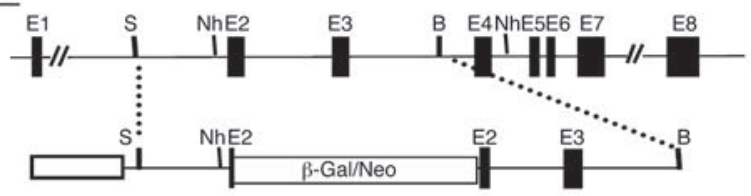

C

Targeted allele $11.5 \mathrm{~kb}$

WT allele $8.5 \mathrm{~kb}$

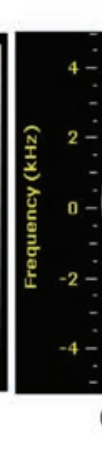

\section{Figure 3}

First-degree AV blockinglobal CAR-KO embryos. (A) Generation of global $C A R$-deficient mice by gene targeting. Exon (E) 2 of $C A R$ was deleted and replaced with a $\beta$-galactosidaseneomycin cassette ( $\beta-\mathrm{Gal} / \mathrm{Neo})$. B, Bglll. (B) Homologous recombination was confirmed by Southern bolt analysis using genomic DNA digested with Nhel from selected ES cells. WT (+/+); heterozygous (+/-). (C) Genotype of the embryos was confirmed by Southern blot analysis on DNA isolated from embryo yolk sacs (left panel). CAR expression was determined using immunoblot analysis of embryos (right panel) of heterozygous, homozygous-deficient $(-/-)$, and WT mice. (D) AV block in global CAR-KO mouse embryos was demonstrated by Doppler analysis of mitral inflow during the period of early rapid filling $(E)$, atrial filling $(A)$, and aortic outflow (OF). The PR intervals were calculated using the time from the beginning of the A-wave to the beginning of the aortic outflow. Mean $\mathrm{PR}$ intervals and heart rate in $C A R^{+/+}$ $(n=8), \mathrm{CAR}^{+-}(n=9)$ and in $C A R^{-/-}$ $(n=8)$ embryos are shown (lower panel). Mean \pm SD. ${ }^{*} P<0.001$.
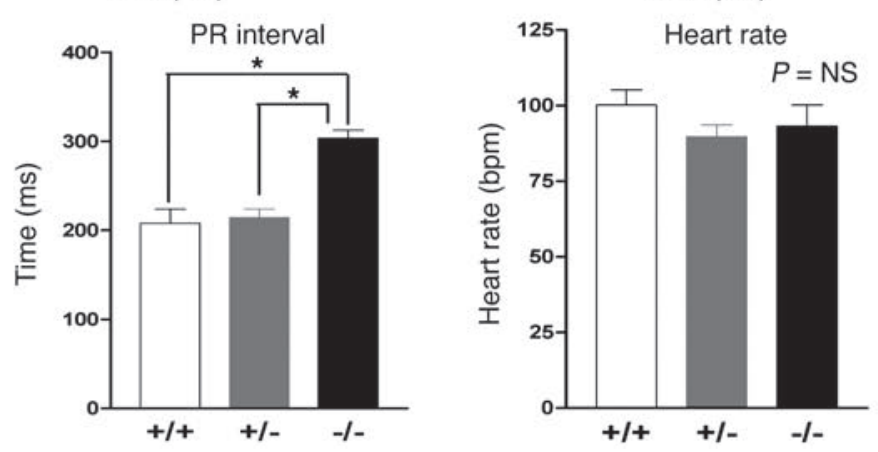

moplakin, and connexin 43 were localized to the intercalated disc in the CAR-cKO mice (Figure 6B). However, there was a marked and consistent decrease in $\beta$-catenin and $\mathrm{ZO}-1$ localization to the intercalated disc of CAR-cKO mouse hearts (Figure 6C). Vinculin staining demonstrated that the intercalated disc was intact but that there was a clear decrease in $\mathrm{ZO}-1$ localized to the intercalated disc (Figure 6C). These results indicate that the absence of CAR affects the level and localization of $\beta$-catenin and $\mathrm{ZO}-1$ while sparing many other intercalated disc proteins. Interestingly, disruption of $\beta$-catenin in the heart has been previously demonstrated to have an effect on cardiac function (17).

Electron microscopic evaluation of the cardiomyocyte and intercalated disc in the absence of CAR. Histologic evaluation at 8 weeks of age was normal by $\mathrm{H} \& \mathrm{E}$ staining (data not shown). Intercalated disc struc- ture was assessed in WT and CAR-cKO mice by electron microscopy at 8 weeks of age. While there were no clear abnormalities in myofilaments, intercalated discs were punctuated by areas where there was an increase in the space between cardiac myocytes. These were generally found in the adherens junctions of the intercalated disc. Concomitant with these abnormalities, there was apparent disorganization in the electron-dense structures associated with the intercalated disc (Figure 6D) and disorganization of the myofilament attachments to the intercalated disc at the same location. Gap junctions were present and appeared intact in the ventricles of both WT and CAR-cKO mice (data not shown).

Late-onset cardiomyopathy in CAR-cKO mice. To determine whether the AV block was associated with ventricular dysfunction and whether the abnormalities in the intercalated discs of the ventricles were asso- 

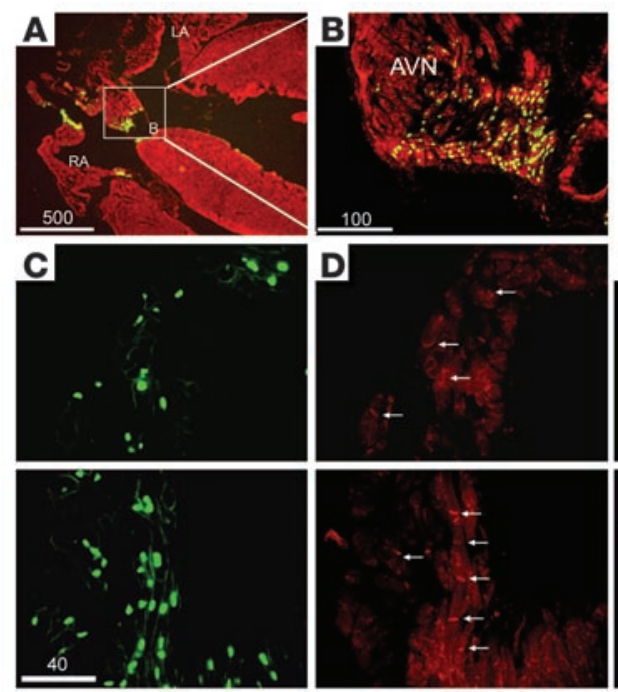

HCN4-GFP

CAR $\mathbf{F}$ WT

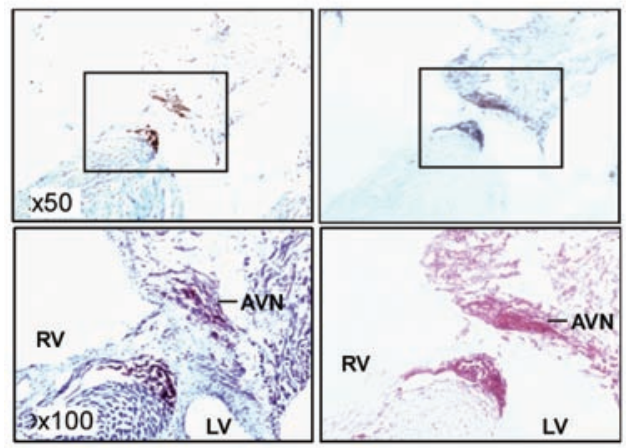

LV

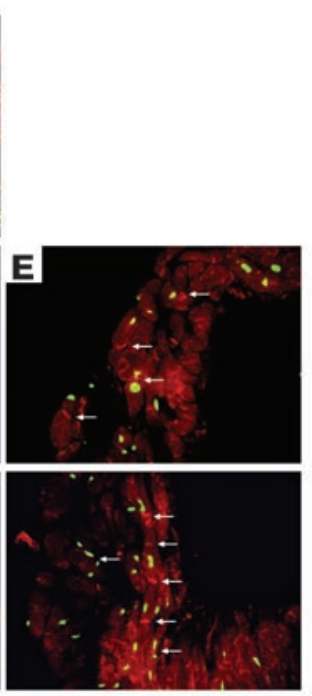

Merge

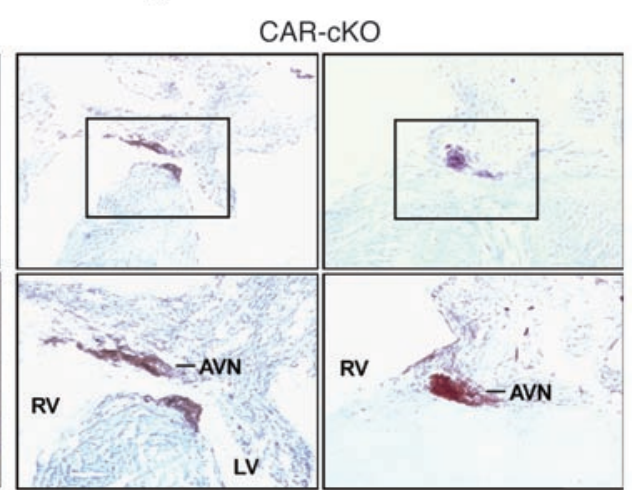

\section{Figure 4}

CAR expression in AV node of WT mice and intact $A V$-node structure in WT and CAR-cKO mice. (A and B) GFP nuclear staining in HCN4-GFP knockin mouse. (A) Section through the right atria (RA) and left atria (LA) shows the AV node in $B$. (B) Higher power view of the AV node (AVN). (C) High-power view from HCN4-GFP knockin mouse (green nuclear stain) at the region of the AV node. Scale bars are measured in micrometers. (D) Immunofluorescent staining for CAR (red, indicated by arrows). (E) Merged images of $\mathbf{D}$ and $\mathbf{E}$ demonstrate that CAR is located between GFP-positive AV-nodal cells (arrows). Lower panels in C, D, and E show different areas from the same mouse. (F) Acetylcholinesterase stain of the WT and CAR-cKO mice demonstrate similar staining patterns in the AV node. Lower panels show enlarged photographs of the insets. ciated with cardiomyopathy, ventricular function was evaluated at several time points. Similar to a previous report (5), cardiac-specific disruption of CAR using the $\alpha$-MHC-CRE transgene did not cause embryonic lethality in the current study. The CAR-cKO mice survived to at least 33 weeks of age without an increase in mortality. Histology and cardiac function was normal at 8 weeks of age, a time point at which AV block and abnormalities in the intercalated discs and intercalated disk proteins had already been detected. However, histologic evaluation of hearts of the CAR-cKO mice at 25 weeks of age demonstrated a clear increase in myocardial fibrosis (Figure 7A).

Ventricular function by echocardiography was not significantly abnormal up to 17 weeks of age; however, there was a progressive decrease in fractional shortening, an echocardiographic parameter of cardiac function, at 21 and 25 weeks of age in the CAR-cKO mice (Figure 7, B and C). There was not a significant increase in LV end-diastolic dimension at 25 weeks of age nor significant differences in heart weight/tibia length (Figure 6C) or heart weight/body weight (not shown) between CAR-cKO and WT mice. This demonstrates that the absence of CAR is associated with a decrease in cardiac function at older ages without a clear increase in ventricular end-diastolic dimension or heart weight to body weight ratios. Therefore, the AV block described occurred in embryos and at a time point that clearly preceded the onset of cardiomyopathy.

CAR forms a complex with connexin $45, \beta$-catenin, and ZO- 1 . The cytoplasmic domains of CAR and connexin 45 have been shown to have PDZ-binding motifs that bind to PDZ domain-containing proteins such as ZO-1 $(8,18,19)$. In order to define the interaction between CAR and connexin 45, whole-heart extracts were immunoprecipitated with CAR antibody. This pulled down connexin 45. Immunoprecipitation with connexin 45 antibody also precipitated CAR (Figure 8A). Connexin 45 is not expressed in HeLa cells. Therefore, a connexin 45-Flag expression vector was coexpressed with a CAR expression vector in HeLa cells. Coimmunoprecipitation using anti-Flag agarose demonstrated that CAR coprecipitated with connexin 45 . However, a mutated CAR (CAR $\triangle \mathrm{PDZ}$ ) expression vector in which the PDZ-binding motif (ITVV) at the C terminus of CAR had been deleted failed to coprecipitate with cotransfected connexin 45-Flag (Figure $8 \mathrm{~B})$. It has been reported previously that the $\mathrm{C}$ terminus of connexin 45 interacts with PDZ domains of ZO-1 (19). To determine whether the PDZ-binding motif of connexin 45 was required for the interaction with CAR, the $4 \mathrm{C}$-terminal residues (SVWI) were mutated to alanine. The mutated connexin 45 no longer interacted with CAR. Cells were also transfected with Flag-tagged 4.5 LIM domain protein 2 (FHL2) as a negative control to demonstrate the lack of interaction with other membrane proteins (Figure 8C) (20). These findings demonstrate that the CAR-connexin 45 protein complex requires the PDZ-binding motif at the $\mathrm{C}$ terminus of both CAR and connexin 45 and indicates that a PDZ domain-containing protein such as $\mathrm{ZO}-1$ is involved in the CAR-connexin 45 protein complex. This is consistent with 
A
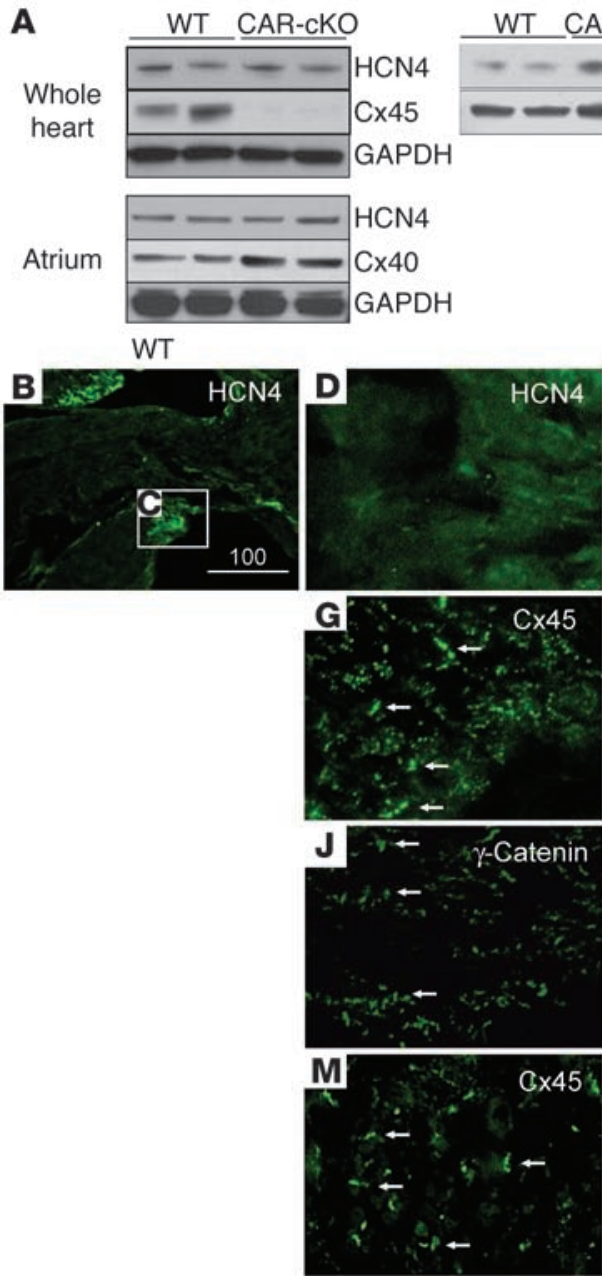

CAR-CKO
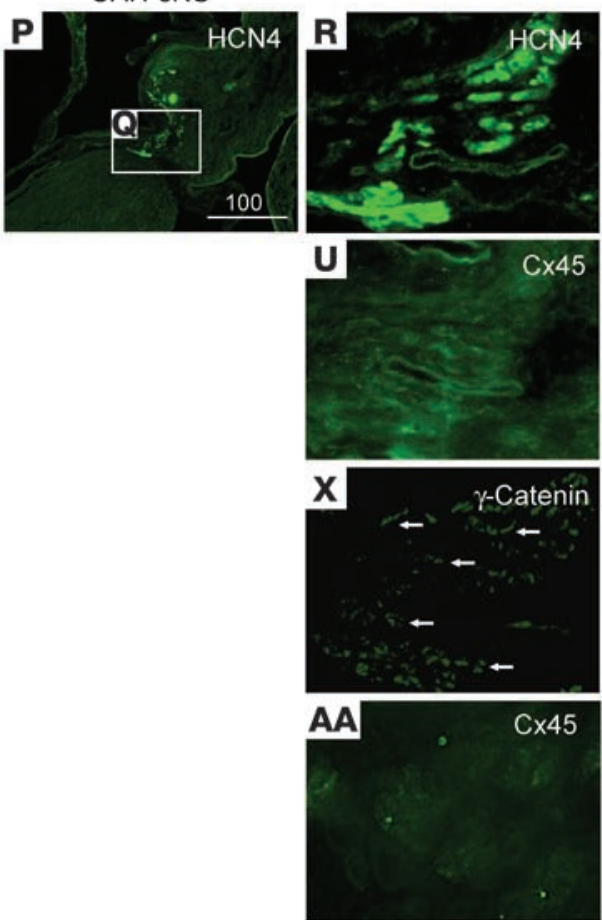
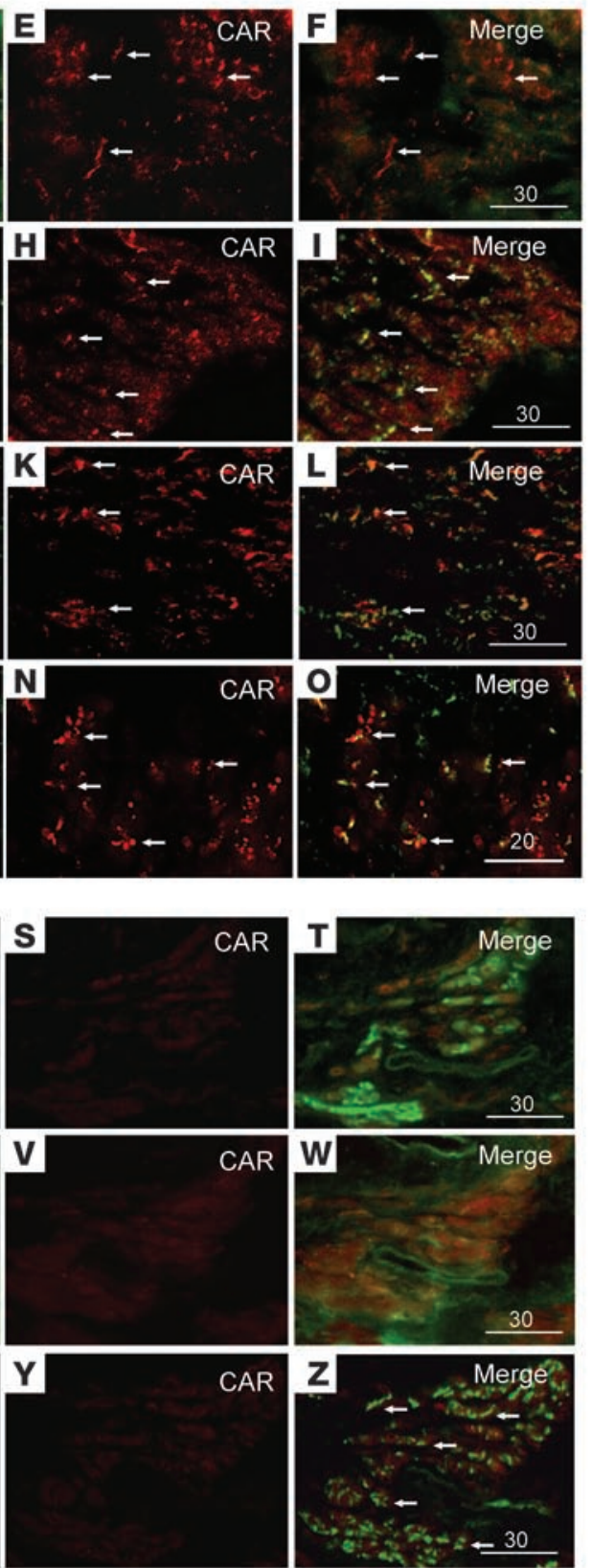

BB
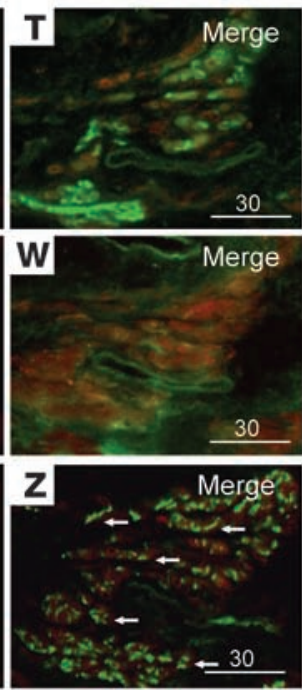

CAR
CC

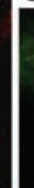

Figure 5

Protein expression in AV node of WT and CAR-cKO mice. (A) Immunoblot for HCN4, connexin 45 (Cx45), connexin 30.2 , and connexin 40 , and GAPDH demonstrate that the levels of connexin 45 are markedly decreased whereas levels of connexins 30.2 and 40 are somewhat increased. (B-CC) Immunofluorescent staining of the AV node in WT (B-O) and CAR-CKO (P-CC) mice. HCN4 stain in the area of the AV node identified the conduction tissue ( $\mathbf{B}$ and $\mathbf{P}$ ). Higher magnification of the area of the AV node (rectangles) stained with $\mathrm{HCN} 4$ and CAR (D-F and R-T). An adjacent section of the AVnodal tissue that stained positive for HCN4 was costained for connexin 45 and CAR (G-I and $\mathbf{U}-\mathbf{W})$. As a control for AV-node, cell-cell junction adjacent sections were costained for $\gamma$-catenin and CAR ( $\mathbf{J}-\mathbf{L}$ and $\mathbf{X}-\mathbf{Z}$ ). The AV nodes of WT and CAR-cKO mice were then costained for CAR and connexin 45 and imaged at high magnification using deconvolution microscopy ( $\mathbf{M}-\mathbf{O}$ and $\mathbf{A A}-\mathbf{C C}$ ). Scale bars are measured in micrometers. Arrows indicate staining of cell-cell junctions. 
A
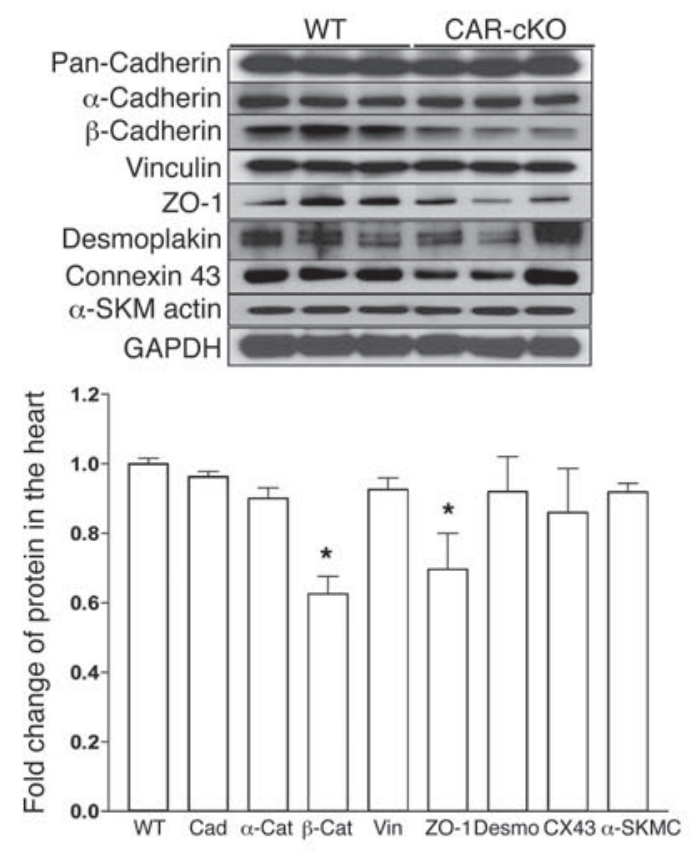

C

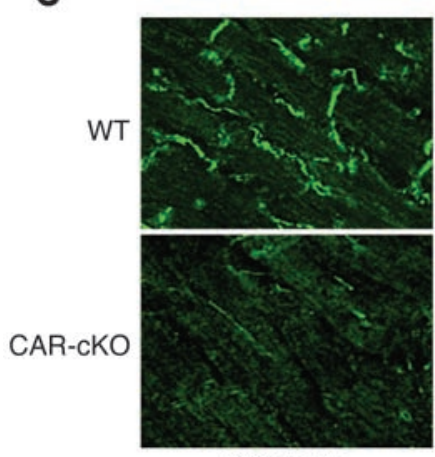

$\beta$-Catenin

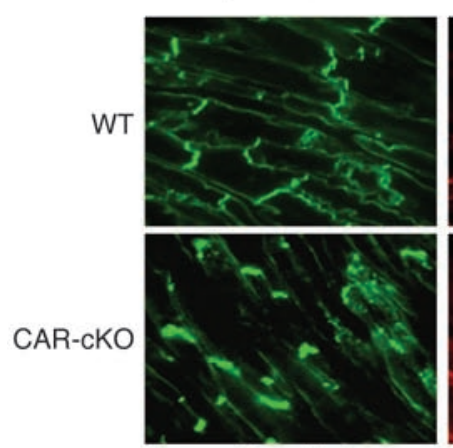

Vinculin

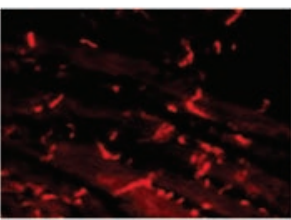

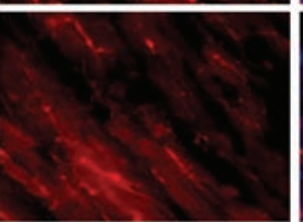

ZO-1

\section{B}

Pan-Cadherin
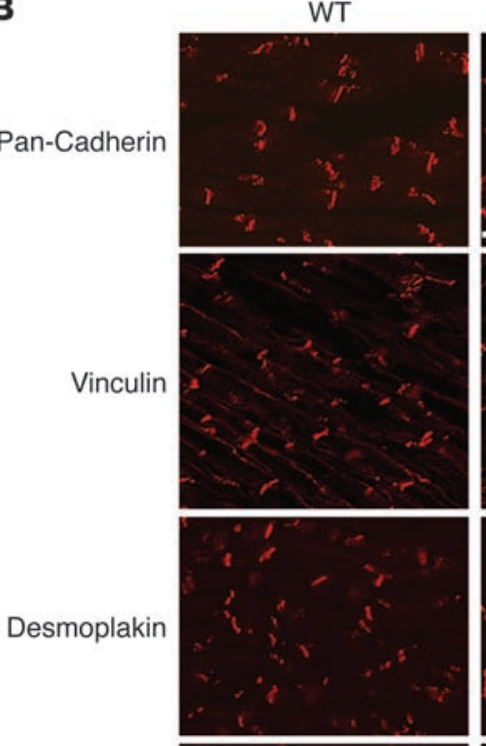

Connexin 43

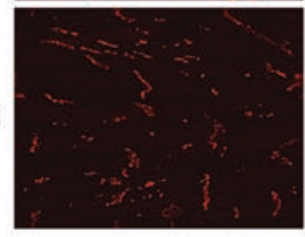

D

WT
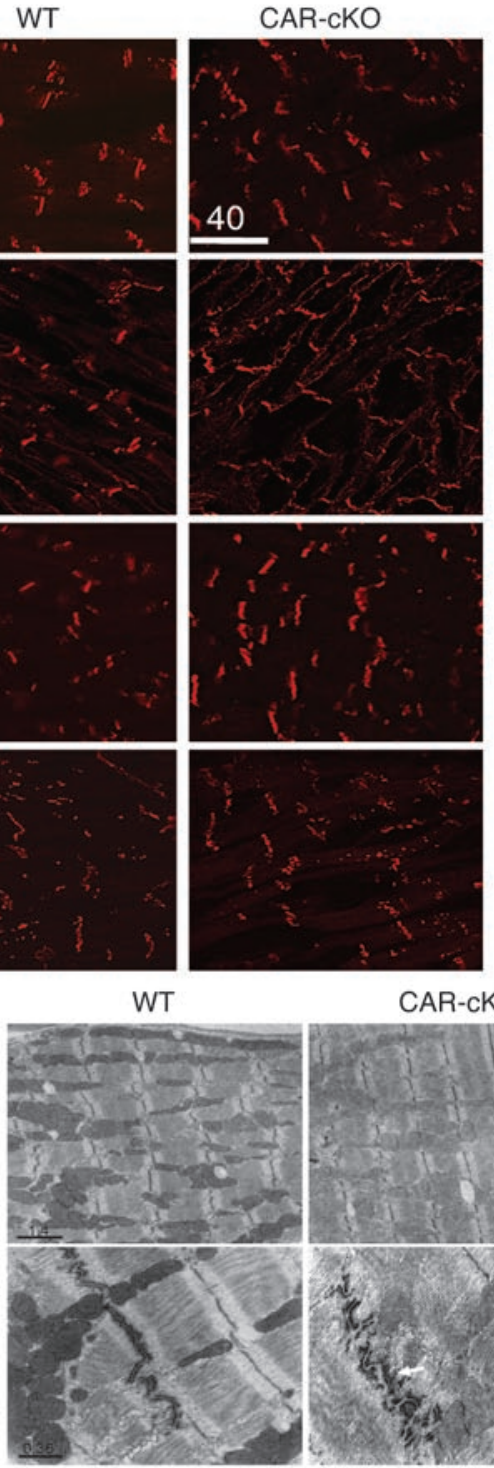

CAR-CKO

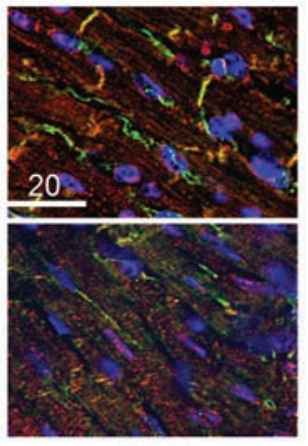

Merge

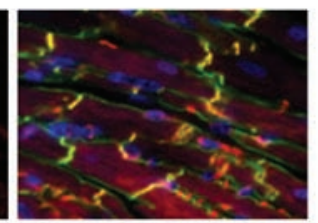

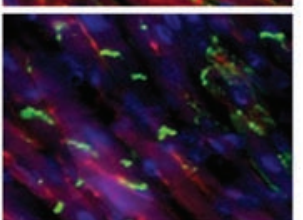

Merge

\section{Figure 6}

Abnormal intercalated disc proteins and cardiomyopathy in CAR-cKO mice. (A) Intercalated disc protein levels in 8-week-old WT and CAR-cKO mouse ventricles by immunoblot. $\beta$-catenin and ZO-1 were significantly reduced in mutants $\left(n=3\right.$ for each group, $\left.{ }^{*} P<0.01\right)$, as quantified by densitometry. (B) Immunofluorescent stains illustrating the localization pattern of intercalated disc proteins. Scale bar: $40 \mu \mathrm{m}$. (C) 8-week-old mouse hearts were costained for $\beta$-catenin (green) and CAR (red) (upper panels), or vinculin (green) and ZO-1 (red) (lower panels). Localization of $\beta$-catenin and ZO-1 at the intercalated disc was abnormal in CAR-cKO mice. Scale bar: $20 \mu \mathrm{m}$. (D) Representative electron micrographs from the ventricular myocardium of WT and CAR-cKO mice at 8 weeks of age. Scale bars: $1.4 \mu \mathrm{m}$ (upper panels); $0.36 \mu \mathrm{m}$ (lower panels). 
A
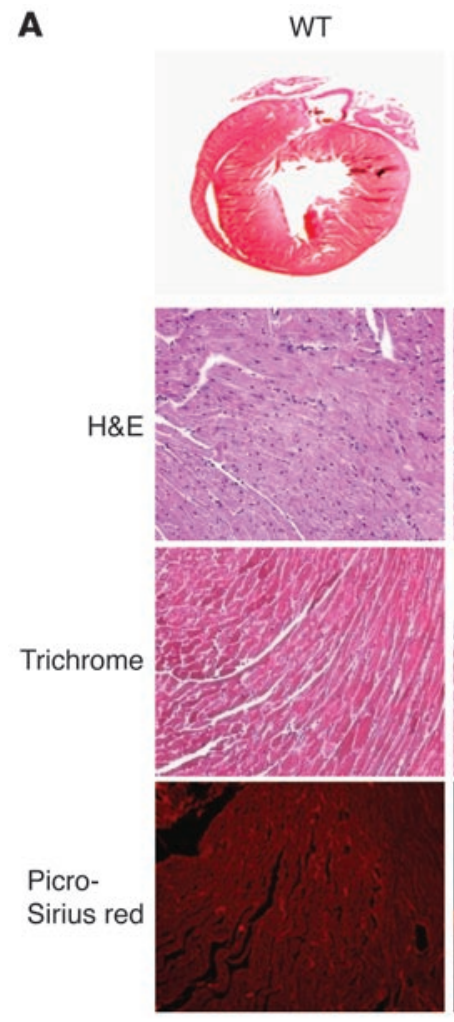

CAR-CKO
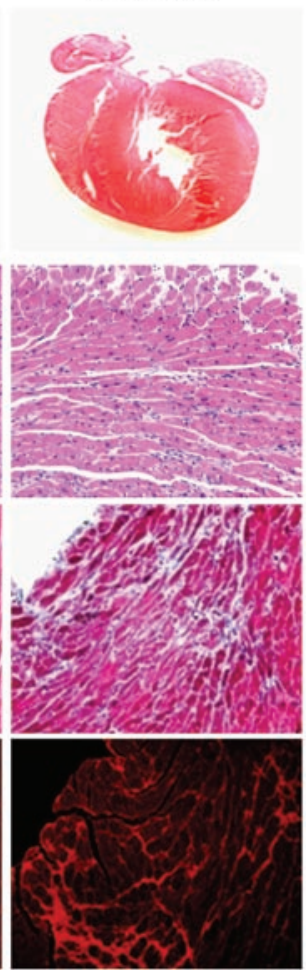

B
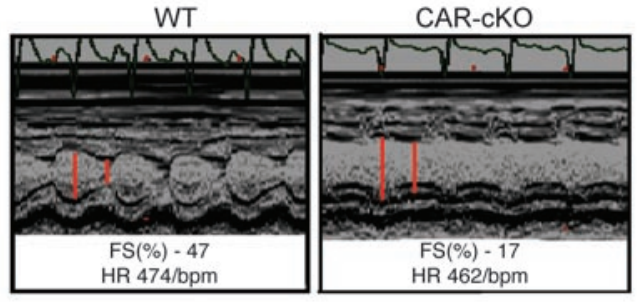

C
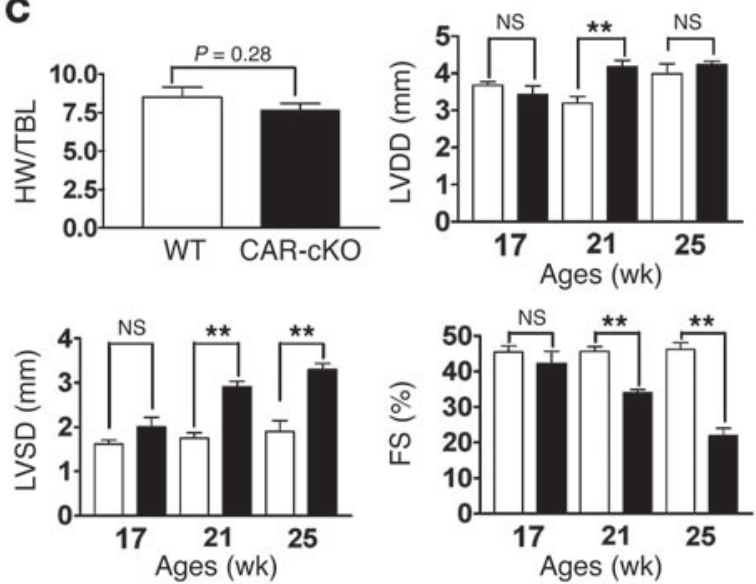

Figure 7

Cardiomyopathy in CAR-cKO mice. (A) Histological findings in WT and CAR-cKO mouse hearts at 25 weeks of age stained with H\&E, trichrome, and picro-sirius red demonstrating myocardial fibrosis. Original magnification, $\times 200$ (lower 6 panels). (B and C) Echocardiographic analysis of CAR-cKO mice showed cardiomyopathy starting around 21 weeks of age (B). The heart weight/tibia length (HW/TBL) ratio was not significantly different between WT and CAR-cKO mice. LV end-diastolic dimension (LVDD), LV end-systolic dimension (LVSD), and fractional shortening (FS) are shown quantitatively $(\mathbf{C}) . n=5$ for each group tested. Error bars show mean \pm SEM. ${ }^{\star \star} P<0.01$ versus WT by Student's $t$ test.

the pull down of ZO-1 in the coprecipitation experiment with full-length CAR. However, we found that connexin 43 does not coprecipitate with CAR (data not shown).

Given the loss of $\beta$-catenin at the intercalated disc of CAR-cKO hearts, we sought to determine whether CAR and $\beta$-catenin may associate in the heart. $\beta$-catenin coprecipitated when immunoprecipitation of ventricular protein extracts was performed with anti-CAR antibody but was not precipitated with IgG as a control (Figure 8D). To define the direct interaction between CAR and $\beta$-catenin, HeLa cells were transfected with CAR-Flag expression vector. Immunoprecipitation with anti- $\beta$-catenin antibody pulled down CAR-Flag (Figure 8E). Immunoprecipitation with anti-Flag agarose eluted with a $3 \mathrm{x}$ Flag peptide demonstrated the presence of $\beta$-catenin in the eluate. This did not occur with transfection of FHL2-Flag as a negative control (Figure 8F). These findings indicate that CAR and $\beta$-catenin are part of a protein complex. However, cotransfection of connexin 45 -Flag expression vector with $\beta$-catenin expression vector failed to demonstrate an interaction between $\beta$-catenin and connexin 45 (data not shown). These results demonstrate that CAR and connexin 45 can associate in a protein complex and that the PDZ-binding motif on CAR and connexin 45 is required for this interaction. While connexin $45, \mathrm{ZO}-1$, and $\beta$-catenin can form a complex with CAR, it appears that connexin 45 and $\beta$-catenin do not have a direct association to each other.

\section{Discussion}

Our findings demonstrate that CAR is required for normal function of the AV node in both the adult and embryonic hearts and for normal ventricular function with aging. Since connexin 45 is a prominent connexin expressed in the compact AV node (21), the loss of connexin 45 in the cell-cell contacts of the AV node provides a sound explanation for why there is abnormal AV conduction in hearts that lack CAR. While the conduction system also expresses connexin 30.2, it appears that it is not sufficient to maintain normal AV conduction. This may be related to its very slow conduction properties (11).

Persistent expression and localization of connexins 40 and 43 in other conduction and myocardial cells likely accounts for the normal conduction in the atria and the ventricles as determined by optical mapping. The AV-node dysfunction identified occurs in the absence of detectable myocardial disease, but it is also important to note that the deficiency of CAR in the heart causes a relatively late onset cardiomyopathy identified at 21 weeks of age. The cardiomyopathy is associated with loss of $\beta$-catenin and ZO- 1 in the cardiomyocyte intercalated disc. Immunoprecipitation experiments combined with the in vivo pathophysiologic effects of CAR disruption indicate that CAR and connexin 45 are part of a previously unrecognized protein complex and that CAR also interacts with $\beta$-catenin and the $\mathrm{PDZ}$ domain-containing protein $\mathrm{ZO}-1$. 
A

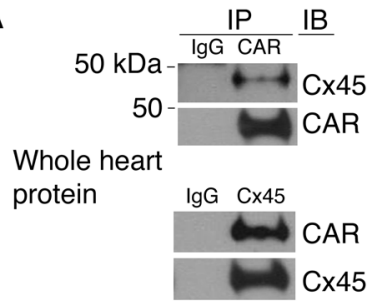

C

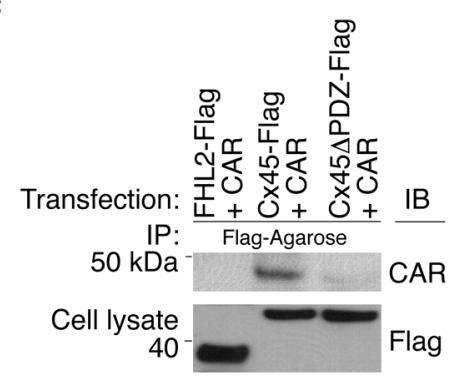

E Transfection: CAR-Flag IB

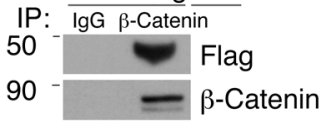

B

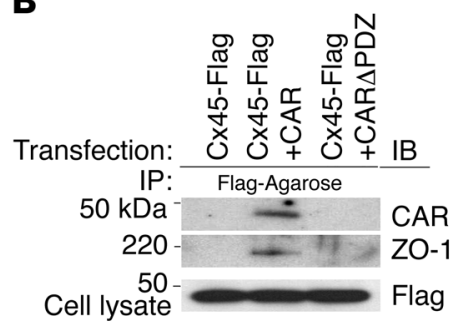

D

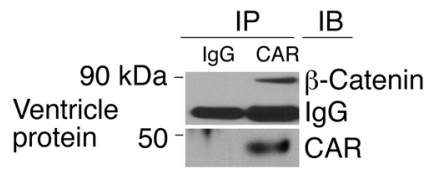

$\mathbf{F}$

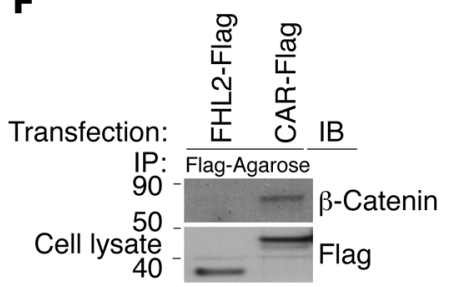

\section{Figure 8}

CAR associates with connexin $45, \beta$-catenin, and ZO-1. (A) Whole-heart protein extracts were immunoprecipitated using IgG as a negative control and anti-CAR (top panels) or connexin 45 antibodies (bottom panels). (B) HeLa cells were transfected with connexin 45-Flag only (Cx45-Flag; lane 1) as control, with connexin 45-Flag and CAR (Cx45-Flag + CAR; lane 2), or with connexin 45-Flag and CAR lacking the PDZ domain-binding motif (Cx45-Flag + CARAPDZ; lane 3). Connexin 45-Flag pulled down CAR and ZO-1 in the connexin 45-Flag- and CARexpressing HeLa cells. In contrast, connexin 45Flag was not able to precipitate CAR or ZO-1 after transfection of HeLa cells with CAR $\triangle P D Z$. (C) To confirm the importance of the PDZ domain-binding motif of connexin 45, CAR was transfected with connexin 45-Flag (Cx45-Flag + CAR; lane 2) or connexin 45-Flag lacking the PDZ domain-binding motif (Cx45 $\Delta$ PDZ-Flag + CAR; lane 3) or FHL2-Flag as control (FHL2-Flag + CAR; lane 1). Connexin 45 lacking the PDZ domain-binding motif did not coprecipitate with CAR. (D) Protein extracts from the ventricular myocardium were immunoprecipitated using IgG as a negative control or the anti-CAR antibody. $\beta$-catenin and CAR were detected in the precipitate. (E) CAR-Flag was precipitated with anti- $\beta$-catenin antibody in CAR-Flag-transfected HeLa cells. (F) $\beta$-catenin was precipitated with CAR-Flag from HeLa cells transfected with CAR-Flag but not with the negative control, FHL2-Flag.
Other adhesion molecules have been shown to have a crucial role in maintaining the integrity of the intercalated disc. For example, the global $\mathrm{KO}$ of $\mathrm{N}$-cadherin, the stereotypical fascia adherens intercalated disc adhesion molecule, results in midgestational embryonic lethality associated with a severe cardiovascular defect (22). Loss of $\mathrm{N}$-cadherin in the adult cardiac myocyte was reported to result in loss of the intercalated disc structure (23-25). This was accompanied by a loss of connexins 43 and $40, \beta$ - and $\alpha$-catenin, and p120. The effects on connexin 45 were not reported. N-cadherin deficiency resulted in inducible atrial and ventricular arrhythmias and a decrease in the conduction velocity in the ventricle. Altogether, these data have been interpreted to indicate that $\mathrm{N}$-cadherin is required for formation of the intercalated disc and that in its absence there is a generalized loss of intercalated disc structure and localization of intercalated disc proteins. This includes normal formation of the gap junction (26). The loss of CAR has an important effect on the intercalated disc, but it is clearly distinct from that observed with $\mathrm{N}$-cadherin deficiency.

While it is clear that $\mathrm{N}$-cadherin interacts with intracellular proteins, it is not clear whether it is part of a connexin-containing protein complex or whether the loss of $\mathrm{N}$-cadherin disrupts the structure of the intercalated disc to the point that connexins can no longer associate with the intercalated disc. Using pulldown experiments and mutational analysis, the current data indicate that CAR is a part of a protein complex that includes connexin 45. The integrity of this interaction requires the PDZbinding motif at the carboxy terminus of CAR. With loss of $\mathrm{CAR}$, there is loss of connexin $45, \mathrm{ZO}-1$, and $\beta$-catenin localization to the cell-cell contacts of the AV node and loss of $\beta$-catenin and ZO-1 to the intercalated disc. Loss of CAR does not result in loss of the intercalated disc structure as has been reported with deficiency of $\mathrm{N}$-cadherin.

Given the localization of CAR to the tight junction of epithelial cells, it might be expected that CAR would be localized to the fascia adherens in the intercalated disc. However, the existence of a protein complex that includes CAR and connexin 45 indicates that CAR may be juxtaposed to or within the gap junction of the AV node. There are data showing that protein localization to the distinct compartments of the intercalated disc are not as clear as in other cell types (27). Another point for consideration is that the AV-nodal cells have a different morphology than myocardial cells and the cell-cell junctions are structurally distinct from those of the contractile myocardial cells (21). Nevertheless, the data indicate that CAR and connexin 45 are part of a protein complex and that CAR expression is required for localization of connexin 45, ZO- 1 , and $\beta$-catenin.

Previous reports have demonstrated that global $\mathrm{KO}$ of connexin 45 leads to embryonic lethality at E9.5-E10.5, a time point that is similar to that of global CAR KO. Prior to death, there is evidence of an AV-conduction abnormality (28). Similarly, a striated muscle-specific connexin $45 \mathrm{KO}$ mouse has abnormal AV conduction. The AV conduction abnormality in the global CAR-KO embryo has similarities to the connexin $45 \mathrm{KO}$ hearts and suggests the possibility that AV-conduction abnormalities could contribute significantly to the embryonic lethality in the absence of CAR as was demonstrated in the cardiac-specific connexin $45 \mathrm{KO}$ mice (29). Differences in the timing of expression during development for the different promoters that have been used to disrupt CAR 
or connexin 45 in the heart may explain why the CAR-cKO mice described herein did not die during embryonic development, while mice in which disruption of CAR was accomplished using the cardiac troponin T-CRE transgene died during development in a manner similar to that of global CAR-KO mice (5).

It was initially unexpected that CAR-cKO mice had AV block without evidence of conduction abnormalities in the atria or ventricle. The data indicate that an impulse arises normally in or near the SA node of the CAR-cKO atria and that it propagates normally to the AV node where the impulse is blocked or delayed. An escape impulse originating in the AV node or His bundle (below the area of block in the AV node) stimulates a normal pattern of depolarization in the ventricle through both the right and left bundle branches. These findings indicate that the cardiac-specific deletion of CAR in the cardiac myocyte does not significantly affect propagation of the electrical impulse in the cell-cell contacts of the HisPurkinje system or the intercalated discs of the cardiac myocytes and that the most significant effect on the electrical conduction through the intercalated discs occurs at the level of the AV node in the CAR-cKO mice. While we did not detect an abnormality in SA-nodal function, this was not evaluated extensively.

The cytoskeletal adaptor protein ZO-1 has been previously shown to bind to the carboxyterminal PDZ-binding motif of CAR $(7,30)$ as well as to the carboxyl terminus of connexin 43 . It has also been shown that $\mathrm{ZO}-1$ colocalizes with $\mathrm{N}$-cadherin. The last 5 amino acids (aa 378-382) of connexin 43 are required for $Z O-1$ binding in vitro when introduced into a connexin 43-deficient MDCK cell line. However, those amino acids are not essential for colocalization of connexin 43 and ZO-1 in a cell membrane (31). That may explain why in the CAR-cKO mice, $\mathrm{ZO}-1$ was disrupted in the intercalated disc but connexin 43 had a similar pattern of localization in the intercalated disc as in the presence of CAR. In addition, it has been previously shown that $\mathrm{KO}$ of connexin 43 did not affect localization of ZO-1 in Rat-1 cells (32). Our findings indicate that the $\mathrm{CAR}$ and $\mathrm{ZO}-1$ protein complex is required to localize connexin 45 to the cell-cell junction of $\mathrm{AV}$-nodal tissue but that they are not required for localization of connexins 40 and 43 in the intercalated disc.

It is notable that there is loss of $\beta$-catenin localization in the intercalated disc of the CAR-deficient hearts. Disruption of $\beta$-catenin has been demonstrated to cause ventricular dysfunction and attenuated hypertrophic response (17). It is not known whether this phenotype is due to the structural effects of the absence of $\beta$-catenin or alterations in $\beta$-catenin signaling. In our results, CAR deficiency in the heart also leads to a decrease in amount and mislocalization of $\beta$-catenin and a cardiomyopathy. These data demonstrate that CAR is required for localization of $\beta$-catenin to the adult heart intercalated disc and its mislocalization may be related to the development of cardiomyopathy. It is not clear why $\beta$-catenin expression and localization were maintained in the global CAR-deficient embryo in a previous report (4).

Altogether these data define a novel protein complex in which an adhesion protein localized to the cell-cell junctions of the AV node and intercalated discs of working myocardium is required for proper localization of connexin $45, \beta$-catenin, and ZO- 1 . CAR is also required for normal AV-node function at early time points and for normal cardiac function as the heart ages. While there are changes noted in the intercalated discs, the effect on the intercalated disc is clearly less than that observed with loss of $\mathrm{N}$-cadherin. These findings demonstrate a clear role for CAR in the heart and have implications for the identification of novel therapeutic and diagnostic strategies for $\mathrm{AV}$ block and ventricular dysfunction in genetic and acquired diseases.

\section{Methods}

Generation of CAR-cKO and global CAR-KO mice. A genomic CAR fragment was isolated from a 129/SVJ library (Stratagene) and used to construct the CAR-targeting vector by standard techniques. For CAR-cKO mice, a floxed CAR construct was generated by inserting loxP sites just before and after the second exon. The targeting vector was digested with SalI and NotI and electroporated into R1 ES cells. G418-resistant ES cells were screened by Southern blot (Figure 1B). Recombination was confirmed using PCR. A positive clone that met all the above criteria was injected into blastocysts from C57/B6 mice. Chimeric mice were inbred with Swiss Black mice to generate germline-transmitted heterozygous mice. CAR genotype was confirmed by PCR (Figure 1C). CAR-cKO mice were compared with mice that were homozygous for the WT genotype of CAR.

For global CAR-KO mice, exon 2 was deleted and replaced by a $\beta$-galactosidase-neomycin selection cassette (Figure $3 \mathrm{~A}$ ). The targeting vector was electroporated into R1 ES cells. One clone was identified that had undergone homologous recombination (Figure $3 \mathrm{~B}$ ). This clone was injected into blastocysts from C57/B6 mice. Germline-transmitted $\mathrm{CAR}^{+/-}$mice were then identified. These heterozygous mice were mated with Swiss Black mice to generate homozygous $C A R^{-/}$embryos. All procedures were carried out in accordance with guidelines set by the UCSD Institutional Animal Care Program and with IACUC approval.

Immunoblot analysis. To evaluate CAR and intercalated disc protein expression level, heart proteins from 8-week-old mice $(n=3)$ were subjected to immunoblot analysis using an ECL detection system as described previously (33). Primary antibodies used were rabbit anti-CAR (Santa Cruz Biotechnology Inc.), pan-cadherin (Sigma-Aldrich), vinculin (Sigma-Aldrich), $\alpha$-catenin (Sigma-Aldrich), $\beta$-catenin (BIOMOL International), ZO-1 (Zymed; Invitrogen), desmoplakin (AbD Serotec), connexins 30.2, 43, 40, and 45 (Zymed; Invitrogen), $\alpha$-skeletal muscle actin (Sigma-Aldrich), and GAPDH (Santa Cruz Biotechnology Inc.). Protein expression levels were evaluated with Image Lab software (MCM Design) based on the signal intensity of each protein using $\alpha$-skeletal muscle actin or GAPDH as an internal control for protein loading.

Expression vectors. $\mathrm{N}$-terminal Flag-tagged CAR and connexin 45-tagged vector were generated for pull-down assays. CAR and connexin 45-Flagtagged vectors were also mutated to eliminate the $\mathrm{C}$-terminal $\mathrm{PDZ}$ binding motifs (ITVV for CAR and SVWI for connexin 45, CAR $\triangle \mathrm{PDZ}$, and connexin $45 \triangle \mathrm{PDZ}$, respectively). The PDZ-binding motif was deleted for CAR, and alanine substitutions were used for connexin 45. Each protein-coding sequence of the mRNA was amplified by RT-PCR from mouse heart and spleen total RNA and cloned into eukaryotic expression vectors using HindIII-EcoRI (for CAR and CAR $\triangle P D Z$ ) and NotI-HindIII (for connexin 45 and connexin $45 \triangle \mathrm{PDZ}$ ) sites. p-3XFlag-CMV-10 (Sigma-Aldrich) and p-EGFP-C3 (Clontech) expression vectors were used for the insertion of $\mathrm{N}$-terminal Flag and GFP tags, respectively.

Coimmunoprecipitation. To examine the direct interaction between CAR and $\beta$-catenin, Flag-tagged CAR protein was expressed in HeLa cells by transfection. The CAR-Flag was pulled down in $500 \mu \mathrm{l}$ of HeLa cell extracts 72 hours after transfection using agarose beads conjugated with anti-Flag (M2) antibody (Sigma-Aldrich). Precipitated agarose beads were washed 4 times with the cell lysis buffer, and the CAR-Flag protein was specifically eluted from the beads by incubation with a 3x Flag peptide (Sigma-Aldrich) using competitive reaction for anti-Flag antibody. The direct binding of CAR to $\beta$-catenin was confirmed using the eluted proteins by immunoblot analysis with anti- $\beta$-catenin antibody. 
To investigate (a) the direct interaction between connexin 45 and CAR and (b) the importance of PDZ-binding motif in CAR and connexin 45 for the interaction, connexin 45-Flag was coexpressed with (a) CAR and (b) CAR $\triangle \mathrm{PDZ}$ or CAR was coexpressed with(a) connexin 45-Flag and (b) connexin $45 \triangle \mathrm{PDZ}$-Flag in the HeLa cells, respectively. As a negative control, FHL2-Flag was coexpressed with CAR. The pull down was performed using anti-Flag agarose beads as described above followed by immunoblot with anti-CAR antibody. FHL2-Flag expression vector was kindly provided by Stephan Lange and Ju Chen (UCSD).

Histology and transmission electron microscopy. H\&E, trichrome, and picrosirius red staining were performed using $10-\mu \mathrm{m}$ paraffin-embedded sections as described previously (34). For transmission electron microscopy, Karnovsky solution, a mixture of $4 \%$ glutaraldehyde and $6 \%$ formaldehyde, was injected into coronary artery by retrograde perfusion to maintain the ultrastructure of the heart. Tissue embedding and processing were performed as described previously (35).

Immunofluorescent staining. Heart was snap-frozen by embedding in OCT Tissue-Tek (Sakura Finetechnical Co. Ltd.) using isobutane chilled in dry ice. $7-\mu \mathrm{m}$ sections were cut by cryosector. Specimens were fixed with ice-cold acetone followed by blocking and permeabilization with $2 \%$ BSA and 1\% Triton X-100 in PBS and incubated with primary antibodies against CAR (1:100; Santa Cruz Biotechnology Inc.), pan-cadherin (1:500; Sigma-Aldrich), vinculin (1:500; Sigma-Aldrich), $\beta$-catenin (1:200; BIOMOL International), ZO-1 (1:200; Zymed, Invitrogen), desmoplakin (1:500; AbD Serotec), connexin 45 (1:50; Zymed, Invitrogen), and HCN4 (1:500, Abcam). The target proteins were visualized with the secondary antibodies conjugated with fluorescence (Alexa Fluor 488 and 594, 1:400; Invitrogen) and Hoechst nuclear stain. Fluorescence images were taken and processed using deconvolution microscope (DeltaVision; Applied Precision). Acethylcholinesterase stain identifying AV node was performed as described previously (36).

Adult and embryo mouse echocardiography. For adult mice, M-mode echocardiograms were performed using 16- to 25 -week-old mice as described previously (35). For embryonic Doppler studies, pregnant mothers were anesthetized with $1 \%$ isoflurane, and the blood flow in the embryonic LV was analyzed using a Vevo 770 Imaging System (VisualSonics). We measured the time from the beginning of the A wave generated by atrial contraction to the beginning of $\mathrm{LV}$ ejection as a substitutive marker for PR interval on ECG to estimate the AV-conduction disturbance. The genotype of each embryo was confirmed by PCR after the Doppler study. Careful recording of the embryo location within the mother by ultrasound made it possible to follow the same embryo over time and to genotype the embryo after the embryos were extracted from the womb.

Surface ECG and optical mapping studies. For ECG study, mice were anesthetized with $1 \%$ isoflurane. Needle electrodes (30 gauge) were inserted subcutaneously into limbs. ECG signals were amplified using Warner Instruments DP-304 Differential Amplifier bandpass filtered between 0.1 and $100 \mathrm{~Hz}$. Signal was further filtered through a Quest Scientific HumBug
$50 / 60 \mathrm{~Hz}$ Noise Eliminator and digitized at $3000 \mathrm{~Hz}$. Data were acquired and analyzed using QRS analysis software (QRS Phenotyping Inc.).

For optical mapping studies, hearts were isolated from 4- to 6-weekold mice ( $n=7$, WT group; $n=6$, CAR-cKO group) and Langendorff perfused with a heated, oxygenated modified Krebs-Henseleit solution. The heart was submersed in heated perfusate in an optical bath chamber, and a volume-conducted ECG was recorded throughout the experiment. The voltage-sensitive dye di-4-ANEPPS ( $1 \mathrm{ml}$ bolus, $26 \mu \mathrm{M})$ was injected into the perfusion line. The atrial or ventricular epicardium was illuminated by $2470 \mathrm{~nm}$ wavelength LED lamps, and fluorescence was filtered at more than $610 \mathrm{~nm}$, focused with a fast video lens (F/0.95; Navitar), and recorded by a 12-bit charge-coupled device camera $(64 \times 64$ pixels, 950 frames/s, CA-D1-0128T; DALSA). During recording of paced beats, the atrium or ventricle was stimulated epicardially with a unipolar platinum electrode at a constant current $(\times 1.5$ threshold $)$ using a digital stimulator (DS8000; WPI). Activation patterns were assessed in the freely beating heart, but $15 \mathrm{mM}$ of the electromechanical uncoupler 2,3-butanedione monoxime was perfused to eliminate motion artifacts while measuring APDs.

Telemetry ECG. For unanesthetized electrocardiography studies, ECG transmitter TA10ETA-F20 or TA10EA-F20 (DSI) was subcutaneously inserted into the backs of the mice as described previously. Positive and negative leads were fixed to the right shoulder muscle and the left leg muscle, respectively. To exclude the effect of injury due to the surgery, data were collected for 24 hours after a 1-week recovery period.

Statistics. Data are expressed as mean \pm SEM unless otherwise noted. Statistical significance was evaluated using the unpaired Student's $t$ test for comparisons between 2 means. For multiple comparisons, 1-way ANOVA using Tukey-Kramer post hoc test was used. $P$ values of less than 0.05 were considered statistically significant.

\section{Acknowledgments}

This project was supported by a grant from the National Heart Lung and Blood Institute of the NIH (to K.U. Knowlton), a Korea Research Foundation grant (Republic of Korea; KRF-2006-214E00012 to B.K. Lim), and American Heart Association grants (to B.K. Lim and D. Xiong).

Received for publication December 17, 2007, and accepted in revised form June 4, 2008.

Address correspondence to: Kirk U. Knowlton, University of California, San Diego, Department of Medicine, 9500 Gilman Drive, La Jolla, California, 92093-0613K, USA. Phone: (858) 822-1364; Fax: (858) 822-3027; E-mail: kknowlton@ucsd.edu.

Byung-Kwan Lim, Dingding Xiong, and Andrea Dorner contributed equally to this work.
1. Bergelson, J.M., et al. 1997. Isolation of a common receptor for Coxsackie B viruses and adenoviruses 2 and 5. Science. 275:1320-1323.

2. Bergelson, J.M., et al. 1998. The murine CAR homolog is a receptor for coxsackie $B$ viruses and adenoviruses. J. Virol. 72:415-419.

3. Asher, D.R., et al. 2005. Coxsackievirus and adenovirus receptor is essential for cardiomyocyte development. Genesis. 42:77-85.

4. Dorner, A.A., et al. 2005. Coxsackievirus-adenovirus receptor (CAR) is essential for early embryonic cardiac development. J. Cell. Sci. 118:3509-3521.

5. Chen, J.W., et al. 2006. Cardiomyocyte-specific deletion of the coxsackievirus and adenovirus receptor results in hyperplasia of the embryonic left ventricle and abnormalities of sinuatrial valves. Circ. Res. 98:923-930.

6. van Raaij, M.J., et al. 2000. Dimeric structure of the coxsackievirus and adenovirus receptor D1 domain at 1.7 A resolution. Structure. 8:1147-1155.

7. Cohen, C.J., et al. 2001. The coxsackievirus and adenovirus receptor is a transmembrane component of the tight junction. Proc. Natl. Acad. Sci. U. S. A. 98:15191-15196.

8. Excoffon, K.J., Hruska-Hageman, A., Klotz, M., Traver, G.L., and Zabner, J. 2004. A role for the PDZ-binding domain of the coxsackie B virus and adenovirus receptor (CAR) in cell adhesion and growth. J. Cell Sci. 117:4401-4409.

9. Kashimura, T., et al. 2004. Spatiotemporal changes of coxsackievirus and adenovirus receptor in rat hearts during postnatal development and in cultured cardiomyocytes of neonatal rat. Virchows Arch. 444:283-292.

10. Desplantez, T., Dupont, E., Severs, N.J., and Weingart, R. 2007. Gap junction channels and cardiac impulse propagation. J. Membr. Biol. 218:13-28.

11. Miquerol, L., et al. 2003. Gap junctional connexins in the developing mouse cardiac conduction system. Novartis Found. Symp. 250:80-98; discussion 98-109, 276-279.

12. Mese, G., Richard, G., and White, T.W. 2007. Gap 
junctions: basic structure and function. J. Invest. Dermatol. 127:2516-2524.

13. Coppen, S.R., Severs, N.J., and Gourdie, R.G. 1999. Connexin45 (alpha 6) expression delineates an extended conduction system in the embryonic and mature rodent heart. Dev. Genet. 24:82-90.

14. Kreuzberg, M.M., Willecke, K., and Bukauskas, F.F. 2006. Connexin-mediated cardiac impulse propagation: connexin 30.2 slows atrioventricular conduction in mouse heart. Trends Cardiovasc. Med. 16:266-272.

15. Agah, R., et al. 1997. Gene recombination in postmitotic cells. Targeted expression of Cre recombinase provokes cardiac-restricted, site-specific rearrangement in adult ventricular muscle in vivo. J. Clin. Invest. 100:169-179.

16. Sun, Y., et al. 2007. Islet 1 is expressed in distinct cardiovascular lineages, including pacemaker and coronary vascular cells. Dev. Biol. 304:286-296.

17. Qu, J., et al. 2007. Cardiac-specific haploinsufficiency of beta-catenin attenuates cardiac hypertrophy but enhances fetal gene expression in response to aortic constriction. J. Mol. Cell. Cardiol. 43:319-326.

18. Coyne, C.B., and Bergelson, J.M. 2005. CAR: a virus receptor within the tight junction. Adv. Drug. Deliv. Rev. 57:869-882.

19. Kausalya, P.J., Reichert, M., and Hunziker, W. 2001. Connexin 45 directly binds to ZO-1 and localizes to the tight junction region in epithelial MDCK cells. FEBS Lett. 505:92-96.

20. Bai, S., et al. 2005. FHL2 inhibits the activated osteoclast in a TRAF6-dependent manner. J. Clin.
Invest. 115:2742-2751.

21. Severs, N.J., et al. 2001. Immunocytochemical analysis of connexin expression in the healthy and diseased cardiovascular system. Microsc. Res. Tech. 52:301-322.

22. Radice, G.L., et al. 1997. Precocious mammary gland development in P-cadherin-deficient mice. J. Cell Biol. 139:1025-1032.

23. Charlton, C.A., Mohler, W.A., Radice, G.L., Hynes, R.O., and Blau, H.M. 1997. Fusion competence of myoblasts rendered genetically null for $\mathrm{N}$-cadherin in culture. J. Cell Biol. 138:331-336.

24. Li, J., et al. 2005. Cardiac-specific loss of N-cadherin leads to alteration in connexins with conduction slowing and arrhythmogenesis. Circ. Res. 97:474-481.

25. Kostetskii, I., et al. 2005. Induced deletion of the Ncadherin gene in the heart leads to dissolution of the intercalated disc structure. Circ. Res. 96:346-354.

26. Zuppinger, C., Eppenberger-Eberhardt, M., and Eppenberger, H.M. 2000. N-Cadherin: structure, function and importance in the formation of new intercalated disc-like cell contacts in cardiomyocytes. Heart Fail. Rev. 5:251-257.

27. Borrmann, C.M., et al. 2006. The area composita of adhering junctions connecting heart muscle cells of vertebrates. II. Colocalizations of desmosomal and fascia adhaerens molecules in the intercalated disk. Eur. J. Cell Biol. 85:469-485.

28. Kumai, M., et al. 2000. Loss of connexin 45 causes a cushion defect in early cardiogenesis. Development. 127:3501-3512.

29. Nishii, K., et al. 2003. Mice lacking connexin 45 con- ditionally in cardiac myocytes display embryonic lethality similar to that of germline knockout mice without endocardial cushion defect. Cell Commun. Adhes. 10:365-369.

30. Coyne, C.B., Voelker, T., Pichla, S.L., and Bergelson, J.M. 2004. The coxsackievirus and adenovirus receptor interacts with the multi-PDZ domain protein-1 (MUPP-1) within the tight junction. J. Biol. Chem. 279:48079-48084.

31. Jin, C., Martyn, K.D., Kurata, W.E., Warn-Cramer, B.J., and Lau, A.F. 2004. Connexin43 PDZ2 binding domain mutants create functional gap junctions and exhibit altered phosphorylation. Cell Commun. Adhes. 11:67-87.

32. van Zeijl, L., et al. 2007. Regulation of connexin 43 gap junctional communication by phosphatidylinositol 4,5-bisphosphate. J. Cell Biol. 177:881-891.

33. Lim, B.K., et al. 2006. Virus receptor trap neutralizes coxsackievirus in experimental murine viral myocarditis. Cardiovasc. Res. 71:517-526.

34. Yajima, T., et al. 2006. Innate defense mechanism against virus infection within the cardiac myocyte requiring gp130-STAT3 signaling. Circulation. 114:2364-2373.

35. Xiong, D., et al. 2007. Inducible cardiac-restricted expression of enteroviral protease $2 \mathrm{~A}$ is sufficient to induce dilated cardiomyopathy. Circulation. 115:94-102.

36. El-Badawi, A., and Schenk, E.A. 1967. Histochemical methods for separate, consecutive and simultaneous demonstration of acetylcholinesterase and norepinephrine in cryostat sections. J. Histochem. Cytochem. 15:580-588. 